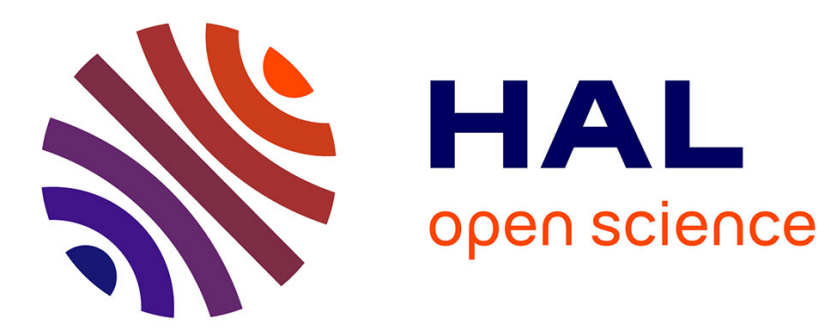

\title{
New Developments and Cosine Effect in the Viscous Shallow Water and Quasi-Geostrophic Equations
}

Carine Lucas, Antoine Rousseau

\section{To cite this version:}

Carine Lucas, Antoine Rousseau. New Developments and Cosine Effect in the Viscous Shallow Water and Quasi-Geostrophic Equations. Multiscale Modeling and Simulation: A SIAM Interdisciplinary Journal, 2008, 7 (2), pp.796-813. 10.1137/070705453 . inria-00392886

\section{HAL Id: inria-00392886 \\ https://hal.inria.fr/inria-00392886}

Submitted on 9 Jun 2009

HAL is a multi-disciplinary open access archive for the deposit and dissemination of scientific research documents, whether they are published or not. The documents may come from teaching and research institutions in France or abroad, or from public or private research centers.
L'archive ouverte pluridisciplinaire HAL, est destinée au dépôt et à la diffusion de documents scientifiques de niveau recherche, publiés ou non, émanant des établissements d'enseignement et de recherche français ou étrangers, des laboratoires publics ou privés. 


\title{
NEW DEVELOPMENTS AND COSINE EFFECT IN THE VISCOUS SHALLOW WATER AND QUASI-GEOSTROPHIC EQUATIONS
}

\author{
C. LUCAS* AND A. ROUSSEAU ${ }^{\dagger}$
}

\begin{abstract}
The viscous Shallow Water Equations and Quasi-Geostrophic Equations are considered in this paper. Some new terms, related to the Coriolis force, are revealed thanks to a rigorous asymptotic analysis. After providing well-posedness arguments for the new models, the authors perform some numerical computations that confirm the role played by the cosine effect in various physical configurations.
\end{abstract}

Key words. multiscale analysis, Coriolis force, cosine effect, asymptotic behavior, shallow water equation, turbulent viscosity, quasi-geostrophic limit, numerical computations.

AMS subject classifications. 76M45, 76U05, 35B40, 35Q35, 76M20.

1. Introduction. In this article, we aim at contributing to the improvement of the derivation of the Shallow Water system. This model, obtained from the incompressible Navier Stokes Equations with free surface under the shallow water approximation, has been studied by numerous authors, both in the inviscid $[20,1]$ and viscous cases $[9,15,2]$. The Shallow Water model has been widely used for theoretical studies and idealized numerical simulations: this is the framework of this article. Conversely, the operational oceanographic research community rather uses the Primitive Equations $([8,14,19])$. But it has to be mentionned that the barotropic part of the Primitive Equations corresponds to the Shallow Water Equations, and carry the most energy (see $[9,21])$. Their study is thus particularly important.

In the theoretical analysis below, we consider a viscosity that is compatible with (physical) numerical computations. Roughly speaking, because of the shape of the domain, the horizontal and vertical resolved eddies give rise to different scales, and this has to be taken into account in the corresponding eddy viscosities; consequently, we will consider an anisotropic turbulent viscosity, as prescribed by [12]. In the ocean, horizontal and vertical eddy viscosities can vary over a wide range (see [17]); whereas [12] choose typical values of the horizontal non-dimensional viscosity of order one, we consider a reasonably smaller value, namely $10^{-3}$. In our physical configuration, the corresponding dimensional value would be $10^{6} \mathrm{~cm}^{2} / \mathrm{s}$.

In the sequel, we derive a new system of equations, in which the above viscosities are taken into account. Simultaneously, in the asymptotic analysis that is classically performed, we show that new terms appear in the so-called viscous Shallow Water Equations (SWE):

$$
\begin{aligned}
& \partial_{t} H+\operatorname{div}_{x}(H u)=0 \\
& \partial_{t}(H u)+\operatorname{div}_{x}(H u \otimes u)+\frac{g}{2} \nabla_{x} H^{2}=-g H \nabla_{x} b-\left(1+\frac{k H}{3 \mu_{V}}\right)^{-1} k u \\
& \quad-\mu_{H} \nabla_{x}\left(H \operatorname{div}_{x} u\right)+2 \mu_{H} \operatorname{div}_{x}\left(H D_{x} u\right)+\boldsymbol{\Omega} \cos \boldsymbol{\theta} \nabla_{\boldsymbol{x}}\left(\boldsymbol{u}_{\mathbf{1}} \boldsymbol{H}^{\mathbf{2}}\right) \\
& \quad+\boldsymbol{\Omega} \cos \boldsymbol{\theta} \boldsymbol{H}^{\mathbf{2}} \boldsymbol{e}_{\mathbf{1}} \operatorname{div}_{\boldsymbol{x}} \boldsymbol{u}-2 \Omega \sin \theta H u^{\perp}-\mathbf{2} \boldsymbol{\Omega} \cos \boldsymbol{\theta} \boldsymbol{H} \boldsymbol{e}_{\mathbf{1}} \boldsymbol{\nabla}_{\boldsymbol{x}} \boldsymbol{b} \cdot \boldsymbol{u}+\mathbf{2} \boldsymbol{\Omega} \cos \boldsymbol{\theta} \boldsymbol{u}_{\mathbf{1}} \boldsymbol{H} \boldsymbol{\nabla}_{\boldsymbol{x}} \boldsymbol{b},
\end{aligned}
$$

${ }^{*}$ Université de Grenoble and INRIA, Laboratoire Jean Kuntzmann, BP 53, 38041 Grenoble Cedex 9, France (Carine.Lucas@imag.fr).

${ }^{\dagger}$ INRIA, Laboratoire Jean Kuntzmann, BP 53, 38041 Grenoble Cedex 9, France (Antoine.Rousseau@inria.fr). 
where $u$ denotes the mean velocity and $H$ the water height, $b$ the topography and $\mu_{V}$, $\mu_{H}$ some eddy viscosities (see below). The angular speed of the Earth is $\Omega, \theta$ is the latitude, and $a$ represents the capillarity, $k$ the friction coefficient.

These new terms (bold-faced in the above equation) are of the order of the viscous terms; they are classically neglected with no rigorous justification. They also arise in the derivation of the viscous Quasi-Geostrophic (QG) Equations:

$$
\begin{array}{r}
D_{t}\left(\left(\partial_{x_{1}}^{2}+\left(1+\boldsymbol{\delta}^{\mathbf{2}}\right) \partial_{x_{2}}^{2}\right) \psi-\frac{\left(2 \Omega \sin \theta_{0}\right)^{2}}{g H_{\text {char }}} \psi+\left(1-\frac{\boldsymbol{H}_{\boldsymbol{c h a r}}}{\left.\mathbf{2 \operatorname { t a n } \boldsymbol { \theta } _ { \mathbf { 0 } }} \boldsymbol{\partial}_{\boldsymbol{x}_{\mathbf{2}}}\right)} \frac{2 \Omega \sin \theta_{0}}{H_{\text {char }}} b+\beta x_{2}\right)\right. \\
=-\frac{1}{\varepsilon L_{\text {char }}} \alpha_{0}\left(H_{\text {char }}\right) \Delta \psi+\mu_{H} \Delta^{2} \psi+\operatorname{curl} f
\end{array}
$$

where $D_{t}=\left(\partial_{t}+u^{0} \cdot \nabla_{x}\right), H_{\text {char }}=\varepsilon L_{\text {char }}$ and $\delta=\Omega \sqrt{H_{\text {char }} / g} \cos \theta_{0}$. The coefficient $\alpha_{0}$ is related to the friction factor.

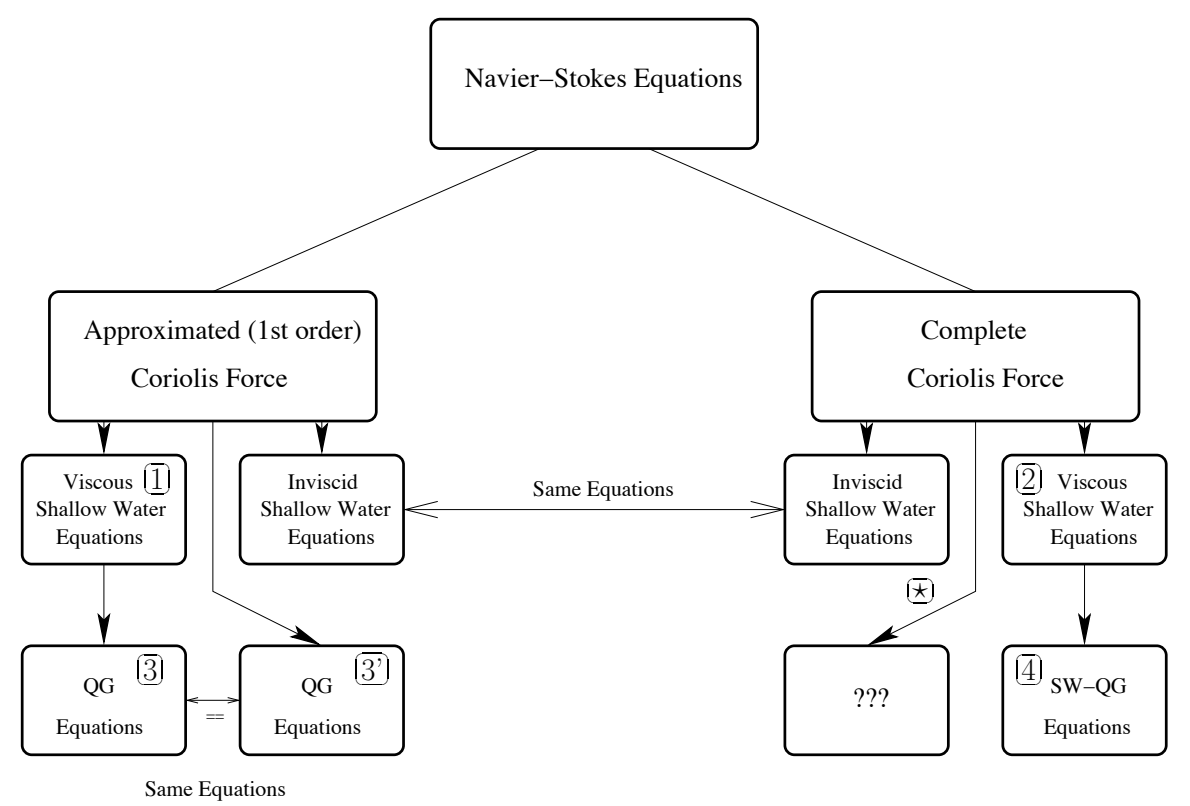

FIG. 1.1. Hierarchy of models.

We present in Figure 1.1 the hierarchy of derivations that have been obtained in the past. The left part corresponds to the classical derivation of SWE by SaintVenant [20], together with the recent works of [9] for the viscous SWE. It is interesting to notice (at least for the rigid lid configuration), that the QG equations are the same $(3=3$ ' in the figure) when derived from the viscous Shallow Water Equations (see [6]) or from the Navier-Stokes equations $([16,11])$. The right part corresponds to the work of [13] and the present article. As Figure 1.1 shows, the inviscid SWE are the same, wether or not the first order approximation is made on the Coriolis force. In other words, the complete Coriolis force does not modify the asymptotic development at the first order. However, and this is the main point of this work, the corresponding viscous equations differ from the one in [9]. Starting from the new SWE and considering the quasi-geostrophic approximation, we end up with another 
model (see box 4 in Figure 1.1). This so-called viscous Quasi-Geostrophic Shallow Water model has been introduced by [4] and is a priori different from the one obtained by $[6]^{1}$.

The aim of this work is to show that the so-called "cosine effect" (see new terms in equations above) has to be considered in the models. In other words, and looking at Figure 1.1, we want to emphasize the differences between models $(3)$ and 4 .

The article is organized as follows. In Section 2, following the ideas of $[9,15,12]$, we perform the asymptotic analysis that leads to the Shallow Water Equations, and then derive the Quasi-Geostrophic limit with cosine effect. In Section 3, we show that the new model is mathematically satisfying, and provide some a priori estimates that ensure well-posedness of the system in the corresponding Sobolev spaces. Finally, we present some numerical simulations that compare the QG model with and without the cosine terms. These simulations show, at least for some realistic situations and as far as the long time behavior is concerned, that this cosine effect has to be taken into account.

2. Derivation of the Two New Models. This section is devoted to the new derivation of the Shallow Water and Quasi-Geostrophic models. First, we show in Section 2.1 that for some choice of the horizontal and vertical viscosities (depending on the aspect ratio) one should consider a complete Coriolis force to get the viscous Shallow Water System. We then obtain a new model from which we can derive, in Section 2.2, the corresponding Quasi-Geostrophic Equations.

2.1. Cosine Effect on Shallow Water Equations. The two dimensional Shallow Water System is obtained from three dimensional Navier-Stokes Equations in a shallow domain. We look for the equations satisfied by the horizontal mean velocity field and the free surface.

We consider 3D Navier-Stokes Equations (NSE) for an homogenous fluid:

$$
\begin{aligned}
& \partial_{t} U+\operatorname{div}(U \otimes U)=\operatorname{div} \sigma-2 \vec{\Omega} \times U+f, \\
& \operatorname{div} U=0,
\end{aligned}
$$

for $(x, z)$ in $\mathbb{T}^{2} \times[b(x), h(t, x)]$, where $U=(u, w) \in \mathbb{R}^{2} \times \mathbb{R}$ is the fluid velocity, $\sigma$ is the stress tensor (given by $-p \mathrm{Id}+S, S$ to be detailed in the sequel), $2 \vec{\Omega} \times U$ is the Coriolis force with $\vec{\Omega}=\Omega(0, \cos \theta, \sin \theta), \theta$ represents the latitude and will be first considered as a constant. Finally $f=-g \overrightarrow{e_{3}}$ is the gravity force.

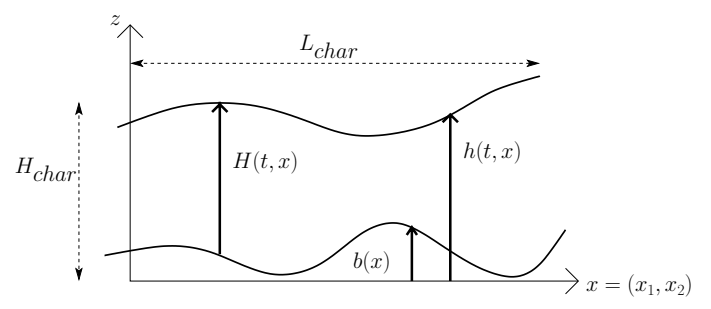

FIG. 2.1. Notations used for the Shallow Water System

\footnotetext{
${ }^{1}$ The direct derivation of the viscous quasi-geostrophic equations starting from the Navier-Stokes equations with a complete Coriolis force (see path $\star$ in Figure 1.1) is still, to the best of our knowledge, an open problem.
} 
Figure 2.1 above describes the computational domain, together with the bathymetry $b$, the water column height $H$ and the free surface $h$. We supplement Equation (2.1) with the following boundary conditions:

- at the free surface $z=h(t, x)$ :

we usually neglect atmospheric pressure and take

$$
\sigma n=0 .
$$

Sometimes we can add surface tension effects, that is

$$
\sigma n=a \kappa n,
$$

where $a$ is the capillarity coefficient, $\kappa$ is the mean curvature, $n$ the unit vector normal to the surface.

But in each case we know that the normal velocity in the referential linked to a particle moving on the surface is zero:

$$
\partial_{t} h+u \cdot \nabla_{x} h=w
$$

- at the bottom $z=b(x)$ :

we have Navier type conditions

$$
(\sigma n) \cdot \tau_{1} \tau_{1}+(\sigma n) \cdot \tau_{2} \tau_{2}=k U \cdot \tau_{1} \tau_{1}+k U \cdot \tau_{2} \tau_{2}
$$

where $k$ is the friction coefficient, $\left(\tau_{1}, \tau_{2}\right)$ is a basis of the tangential surface. We add the non-penetration condition

$$
-u \cdot \nabla_{x} b+w=0
$$

In what follows we write these equations under their non-dimensional form, make an asymptotic development of $U$ and study its first orders for a specific choice of turbulent viscosities. Shallow Water Equations are obtained after integrating the first momentum equation over the water height. We also give the Shallow Water System when the latitude is not constant.

Dimensionless Navier-Stokes Equations. We write the Navier-Stokes system and the boundary conditions in a non-dimensionalized form, using some characteristic scales specially chosen to get the Shallow Water model.

Let us start with the 3D NSE for an homogenous fluid. In the following, the subscript $x$ denotes horizontal variables, $u_{1}$ the first component of the vector $u$ and $e_{1}$ the unit vector $(1,0)$. The stress tensor reads $\sigma=-p \mathrm{Id}+S=-p \mathrm{Id}+\mu_{H} D^{H}+\mu_{V} D^{V}+\mu_{E} D^{E}$. In this relation, $p$ is the pressure, $\mu_{H}, \mu_{V}$ and $\mu_{E}$ represents eddy viscosities. More precisely, if $\Lambda$ is the vector :

$$
\Lambda=\gamma(\xi)\left(\begin{array}{l}
\xi \\
1
\end{array}\right) \quad \text { where } \quad \xi=\frac{h-z}{b-h} \nabla_{x} b+\frac{z-b}{b-h} \nabla_{x} h \quad \text { and } \quad \gamma(\xi)=\frac{1}{\sqrt{1+|\xi|^{2}}},
$$

then $\mu_{H}$ denotes the eddy viscosity related to the shear in the direction orthogonal to $\Lambda, \mu_{V}$ the viscosity linked to the shear in the direction of $\Lambda$, and $\mu_{E}$ can be interpreted as the compression rate in the direction of $\Lambda$ (or expansion in the orthogonal direction $)^{2}$. Lastly, the symmetric part of the gradient of $U$ is split as:

$$
2 D(U)=\left(\begin{array}{cc}
\nabla_{x} u+{ }^{t} \nabla_{x} u & \partial_{z} u+\nabla_{x} w \\
{ }^{t}\left(\partial_{z} u+\nabla_{x} w\right) & 2 \partial_{z} w
\end{array}\right)=D^{H}+D^{V}+D^{E},
$$

\footnotetext{
${ }^{2}$ Note that, for a flat bottom, $\Lambda$ corresponds to the vertical direction, hence the subscripts $H$ and $V$ (vertical and horizontal shear).
} 
where the tensors are given by:

$$
\begin{aligned}
& D^{H}=2\left(\operatorname{Id}-\Lambda^{t} \Lambda\right) D(U)\left(\operatorname{Id}-\Lambda^{t} \Lambda\right)+{ }^{t} \Lambda D(U) \Lambda\left(\operatorname{Id}-\Lambda^{t} \Lambda\right), \\
& D^{V}=2\left(\operatorname{Id}-\Lambda^{t} \Lambda\right) D(U) \Lambda{ }^{t} \Lambda+2 \Lambda{ }^{t} \Lambda D(U)\left(\operatorname{Id}-\Lambda^{t} \Lambda\right) \\
& D^{E}={ }^{t} \Lambda D(U) \Lambda\left(3 \Lambda{ }^{t} \Lambda-\operatorname{Id}\right) .
\end{aligned}
$$

Then, the Navier-Stokes system reads:

$$
\begin{aligned}
& \partial_{t} u+u \cdot \nabla_{x} u+w \partial_{z} u=-\nabla_{x} p+\operatorname{div}_{x} S_{x x}+\partial_{z} S_{x z}-2 \Omega \sin \theta u^{\perp}-2 \Omega \cos \theta w e_{1} \\
& \partial_{t} w+u \cdot \nabla_{x} w+w \partial_{z} w=-\partial_{z} p+\operatorname{div}_{x} S_{z x}+\partial_{z} S_{z z}+2 \Omega \cos \theta u_{1}-g \\
& \operatorname{div}_{x} u+\partial_{z} w=0
\end{aligned}
$$

where the tensor $S$ is written as: $S=\left(\begin{array}{cc}S_{x x} & S_{x z} \\ S_{z x} & S_{z z}\end{array}\right)$.

We introduce the following dimensionless variables and numbers:

$$
\begin{aligned}
& x=L_{\text {char }} x^{\prime}, \quad z=H_{\text {char }} z^{\prime}, \quad \text { with } \varepsilon=\frac{H_{\text {char }}}{L_{\text {char }}} \ll 1, \\
& u=u_{\text {char }} u^{\prime}, \quad w=w_{\text {char }} w^{\prime}, \quad \text { with } w_{\text {char }}=\varepsilon u_{\text {char }}, \\
& t=\frac{L_{c a r}}{u_{\text {car }}} t^{\prime}, \quad \quad p=p_{\text {car }} p^{\prime}, \quad \text { with } p_{\text {car }}=u_{\text {car }}^{2}, \\
& \mu_{H}=\varepsilon L_{c a r} u_{c a r} \nu_{H}, \quad \mu_{V}=\varepsilon L_{c a r} u_{c a r} \nu_{V}, \quad \mu_{E}=\varepsilon^{3} L_{c a r} u_{c a r} \nu_{E}, \\
& S_{x x}=u_{c a r}^{2} \varepsilon S_{x x}^{\prime}, \quad S_{x z}=u_{c a r}^{2} S_{x z}^{\prime}, \quad S_{z x}=u_{c a r}^{2} S_{z x}^{\prime}, \\
& S_{z z}=u_{c a r}^{2} \varepsilon S_{z z}^{\prime}, \quad S=u_{c a r}^{2} \varepsilon S^{\prime}, \quad \text { with } S^{\prime}=\left(\begin{array}{cc}
S_{x x}^{\prime} & \frac{1}{\varepsilon} S_{x z}^{\prime} \\
\frac{1}{\varepsilon} S_{z x}^{\prime} & \varepsilon S_{z z}
\end{array}\right) \text {, } \\
& \xi=\varepsilon \xi^{\prime}, \quad \text { Ro }=\frac{u_{c a r}}{2 L_{c a r} \Omega}, \quad F r=\frac{u_{c a r}}{\sqrt{g H_{c a r}}} .
\end{aligned}
$$

where $\varepsilon$ is the aspect ratio, $R o$ the Rossby number, $F r$ the Froude number. We drop the primes to rewrite the 3D NSE:

$$
\begin{aligned}
& \partial_{t} u+u \cdot \nabla_{x} u+w \partial_{z} u=-\nabla_{x} p+\varepsilon \operatorname{div}_{x} S_{x x}+\frac{1}{\varepsilon} \partial_{z} S_{x z}-\frac{\sin \theta}{R o} u^{\perp}-\varepsilon \frac{\cos \theta}{R o} w e_{1} \\
& \partial_{t} w+u \cdot \nabla_{x} w+w \partial_{z} w=-\frac{1}{\varepsilon^{2}} \partial_{z} p+\frac{1}{\varepsilon} \operatorname{div}_{x} S_{z x}+\frac{1}{\varepsilon} \partial_{z} S_{z z}+\frac{1}{\varepsilon} \frac{\cos \theta}{R o} u_{1}-\frac{1}{\varepsilon^{2} F r^{2}} \\
& \operatorname{div}_{x} u+\partial_{z} w=0 .
\end{aligned}
$$

The tensor $S$ is now given by: $S=\nu_{H} D^{H}+\nu_{V} D^{V}+\varepsilon^{2} \nu_{E} D^{E}$ where $D^{H}, D^{V}$ and $D^{E}$ still have the expressions (2.2) but with:

$$
\Lambda=\gamma(\varepsilon \xi)\left(\begin{array}{c}
\varepsilon \xi \\
1
\end{array}\right) \quad \text { and } \quad 2 D(U)=\left(\begin{array}{cc}
\nabla_{x} u+{ }^{t} \nabla_{x} u & \frac{1}{\varepsilon} \partial_{z} u+\varepsilon \nabla_{x} w \\
t\left(\frac{1}{\varepsilon} \partial_{z} u+\varepsilon \nabla_{x} w\right) & 2 \partial_{z} w
\end{array}\right)
$$

We also have to change the boundary conditions. We first replace $\sigma$ with its expression and change the variables:

- at the free surface $z=h(t, x)$ :

the normal vector $n$ is $n=\left(1+\left(\nabla_{x} h\right)^{2}\right)^{-\frac{1}{2}}\left(\begin{array}{c}-\nabla_{x} h \\ 1\end{array}\right)$. The horizontal variable 
$h$ is rescaled as $h=H_{\text {char }} h^{\prime}$ to get the dimensionless conditions at the free surface:

$$
\begin{aligned}
& p \nabla_{x} h-\varepsilon S_{x x} \nabla_{x} h+\frac{1}{\varepsilon} S_{x z}=0, \\
& p-\varepsilon S_{z z}+\varepsilon S_{z x} \nabla_{x} h=0 \\
& \partial_{t} h+u \cdot \nabla_{x} h=w .
\end{aligned}
$$

- at the bottom $z=b(x)$ :

if we write $b=H_{c h a r} b^{\prime}$, the non-penetration condition reads

$$
-u \cdot \nabla_{x} b+w=0
$$

The Navier condition is more involved. We choose the tangential vectors

$$
\tau_{1}=\frac{1}{\left|\nabla_{x} b\right|}\left(\begin{array}{c}
\nabla_{x}^{\perp} b \\
0
\end{array}\right) \text { and } \tau_{2}=\frac{1}{\sqrt{\left|\nabla_{x} b\right|^{2}+\left|\nabla_{x} b\right|^{4}}}\left(\begin{array}{c}
-\nabla_{x} b \\
-\left|\nabla_{x} b\right|^{2}
\end{array}\right) .
$$

We define $K=k u_{c h a r}^{-1} \varepsilon^{-1}$ and obtain a complex expression that we do not detail here. We perform some approximations in the following and give a simplified equality obtained from this expression.

Hydrostatic Approximation. We now use the hydrostatic approximation, that is we suppose the aspect ratio $\varepsilon$ to be small. We only keep the first two orders in Equation (2.3b) and we also drop terms in boundary conditions.

We are led to study the system:

$\partial_{t} u+u \cdot \nabla_{x} u+w \partial_{z} u=-\nabla_{x} p+\varepsilon \operatorname{div}_{x} S_{x x}+\frac{1}{\varepsilon} \partial_{z} S_{x z}-\frac{\sin \theta}{R o} u^{\perp}-\varepsilon \frac{\cos \theta}{R o} w e_{1}$,

$\partial_{z} p=\varepsilon \operatorname{div}_{x} S_{z x}+\varepsilon \partial_{z} S_{z z}-\frac{1}{F r^{2}}+\varepsilon \frac{\cos \theta}{R o} u_{1}$,

$\operatorname{div}_{x} u+\partial_{z} w=0$.

We can simplify the Navier condition

$$
\varepsilon S_{x x} \nabla_{x} b-\frac{1}{\varepsilon} S_{x z}=-K u+O\left(\varepsilon^{2}\right) .
$$

At the free surface, Equation (2.4c) is not modified but we can rewrite (2.4a) as follows:

$$
\varepsilon S_{x z}=-\varepsilon^{2}\left(p \nabla_{x} h-\varepsilon S_{x x} \nabla_{x} h\right)=\varepsilon^{t} S_{z x}+O\left(\varepsilon^{2}\right) \quad \text { for } z=h(x, t),
$$

and plug it into Equation (2.4b):

$$
\begin{aligned}
p-\varepsilon S_{z z} & =-\varepsilon S_{z x} \nabla_{x} h=-\varepsilon S_{x z} \cdot \nabla_{x} h+O\left(\varepsilon^{2}\right) \\
& =\varepsilon^{2}\left(p \nabla_{x} h-\varepsilon S_{x x} \nabla_{x} h\right) \cdot \nabla_{x} h+O\left(\varepsilon^{2}\right), \\
p-\varepsilon S_{z z} & =O\left(\varepsilon^{2}\right), \quad \text { for } z=h(x, t) .
\end{aligned}
$$

We integrate Equation (2.6b) from $h$ to $z$, with $z$ between $b$ and $h$. The value of $p$ at the free surface is given by $(2.8)$, and we find the pressure at order $\varepsilon$ :

$$
p(t, x, z)=\frac{1}{F r^{2}}(h(t, x)-z)+\varepsilon \int_{h}^{z} \operatorname{div}_{x} S_{x x}+\varepsilon S_{z z}+\varepsilon \frac{\cos \theta}{R o} \int_{h}^{z} u_{1}+O\left(\varepsilon^{2}\right) .
$$


As we are looking for equations on the mean velocity and on the evolution of the free surface, we first integrate the momentum equation (2.6a) over the water height (between $z=b(x)$ and $z=h(t, x))$. We apply Leibniz formula and get:

$$
\begin{array}{rl}
\partial_{t} \int_{b}^{h} & u-\partial_{t} h u_{\left.\right|_{z=h}}+\operatorname{div}_{x} \int_{b}^{h}(u \otimes u)-\left(\left(u \cdot \nabla_{x} h\right) u\right)_{\left.\right|_{z=h}}+\left(\left(u \cdot \nabla_{x} b\right) u\right)_{\left.\right|_{z=b}} \\
& +(u w)_{\left.\right|_{z=h}}-(u w)_{\left.\right|_{z=b}}+\nabla_{x} \int_{b}^{h} p=\nabla_{x} h p_{\left.\right|_{z=h}}-\nabla_{x} b p_{\left.\right|_{z=b}} \\
& +\varepsilon \operatorname{div}_{x} \int_{b}^{h} S_{x x}-\varepsilon S_{\left.x x\right|_{z=h}} \nabla_{x} h+\varepsilon S_{\left.x x\right|_{z=b}} \nabla_{x} b+\frac{1}{\varepsilon} S_{\left.x z\right|_{z=h}} \\
& -\frac{1}{\varepsilon} S_{\left.x z\right|_{z=b}}-\frac{\sin \theta}{R o} \int_{b}^{h} u^{\perp}-\varepsilon \frac{\cos \theta}{R o} \int_{b}^{h} w e_{1} .
\end{array}
$$

Then, we use boundary conditions $(2.4 \mathrm{a}),(2.4 \mathrm{c}),(2.5)$ and $(2.7)$ to simplify the expressions at the surface and at the bottom and obtain the integrated momentum equation:

$$
\begin{aligned}
\partial_{t} \int_{b}^{h} u+\operatorname{div}_{x} \int_{b}^{h}(u \otimes u) & +\nabla_{x} \int_{b}^{h} p=-\nabla_{x} b p_{\left.\right|_{z=b}}-K u_{\left.\right|_{z=b}} \\
& +\varepsilon \operatorname{div}_{x} \int_{b}^{h} S_{x x}-\frac{\sin \theta}{R o} \int_{b}^{h} u^{\perp}-\varepsilon \frac{\cos \theta}{R o} \int_{b}^{h} w e_{1}
\end{aligned}
$$

with a new Coriolis term (the last one).

We also want the evolution of the free surface: we integrate the divergence free equation (2.6c) from the bottom to the surface, use Leibniz formula again and conditions surface and bottom $(2.4 \mathrm{c})$ and $(2.5)$ to find:

$$
\partial_{t} h(t, x)+\operatorname{div}_{x} \int_{b(x)}^{h(t, x)} u=0 .
$$

In the sequel we study the integrated momentum equation (2.10) and the free surface equation (2.11) when we approximate $u$ at the first order and at the second order.

Shallow Water System. We have already done the main assumption to get the Shallow Water System, that is the depth is small compared to the length of the domain. Now we develop $u, w, H, p, b$ in powers of $\varepsilon$, that is $u=u^{0}+\varepsilon u^{1}+\varepsilon^{2} u^{2}+\ldots$ (and so on) with $H(t, x)=h(t, x)-b(x)$.

We look for $u^{0}$, the first order of the velocity. We use the horizontal momentum equation (2.6a) and the boundary conditions (2.4a) and (2.7). We obtain

$$
\partial_{z}^{2} u=O(\varepsilon), \quad\left(\partial_{z} u\right)_{\left.\right|_{z=b}}=O(\varepsilon), \quad\left(\partial_{z} u\right)_{\left.\right|_{z=h}}=O(\varepsilon)
$$

which means that at the first order $u$ does not depend on $z: u^{0}(t, x, z)=u^{0}(t, x)$. As we are looking for the dynamics of $u^{0}$, we study the previous equations at the first order. Let us first rewrite the evolution equation (2.11)

$$
\partial_{t} H^{0}+\operatorname{div}_{x}\left(H^{0} u^{0}\right)=0 .
$$

Then we have $p$ at the first order with (2.9):

$$
p^{0}(t, x, z)=\operatorname{Fr}^{-2}(h-z) .
$$


We replace this value in the integrated momentum equation (2.10) and obtain:

$$
\begin{aligned}
\partial_{t}\left(H^{0} u^{0}\right)+\operatorname{div}_{x}\left(H^{0} u^{0} \otimes u^{0}\right)+\frac{1}{2 F r^{2}} & \nabla_{x}\left(H^{0}\right)^{2}= \\
& -\frac{1}{F r^{2}} H^{0} \nabla_{x} b^{0}-K u^{0}-\frac{\sin \theta}{R o} H^{0} u^{0^{\perp}} .
\end{aligned}
$$

Equations (2.12) - (2.13) form the Shallow Water System at the first order in nondimensional variables.

If we go back to dimensional variables we have the Shallow Water System at the first order for a viscosity of order $\varepsilon$ :

$$
\begin{aligned}
& \partial_{t} H+\operatorname{div}_{x}(H u)=0, \\
& \partial_{t}(H u)+\operatorname{div}_{x}(H u \otimes u)+\frac{g}{2} \nabla_{x} H^{2}=-g H \nabla_{x} b-k u-2 \Omega \sin \theta H u^{\perp} .
\end{aligned}
$$

At this point, we get the inviscid Shallow Water system. As underlined in Figure 1.1, the cosine part of the Coriolis force does not modify these equations at the first order. But there are no viscosity effects in this system: we are led to study the second order to make viscous terms appear in our Shallow Water System.

We will denote by a bold letter the approximation of the variables at order $\varepsilon$ (for example, $\boldsymbol{u}^{\mathbf{1}}=u^{0}+\varepsilon u^{1}$ ) and by a bar the mean value on the water height defined by:

$$
\bar{u}(t, x):=\frac{1}{H(t, x)} \int_{b}^{h} u d z .
$$

Let us rewrite the divergence condition at the second order

$$
\partial_{t} \boldsymbol{H}^{\mathbf{1}}+\operatorname{div}_{x}\left(\boldsymbol{H}^{\mathbf{1}} \overline{\mathbf{u}}^{\mathbf{1}}\right)=O\left(\varepsilon^{2}\right)
$$

As before, we rewrite the momentum equation (2.6a) but at order $\varepsilon$, and with the Shallow Water System at the first order (2.12) - (2.13) we obtain an expression for the second derivative of $u$ :

$$
\frac{\nu_{V}}{\varepsilon} \partial_{z}^{2} u=-\frac{K}{H^{0}} u^{0}+O(\varepsilon)
$$

We integrate this equality from $b$ to $z$ (for $z$ between $b(x)$ and $h(t, x)$ ) with the boundary condition (2.7). We integrate it again from $b$ to $z$ to find an approximation of $u$ at the second order:

$$
\begin{aligned}
u & =\boldsymbol{u}_{\left.\right|_{z=b}}+\varepsilon \frac{K}{\nu_{V}} u^{0} \int_{b}^{z}\left(1-\frac{s-b^{0}}{H^{0}}\right) d s+O\left(\varepsilon^{2}\right) \\
& =\boldsymbol{u}_{\left.\right|_{z=b}}\left(1+\varepsilon \frac{K}{\nu_{V}}\left(z-b^{0}\right)\left(1-\frac{z-b^{0}}{2 H^{0}}\right)\right)+O\left(\varepsilon^{2}\right) .
\end{aligned}
$$

With this expression, we can compute the mean value of $u$ :

$$
\bar{u}=\boldsymbol{u}_{\left.\right|_{z=b}}\left(1+\varepsilon \frac{K}{\nu_{V}} \frac{H^{0}}{3}\right)+O\left(\varepsilon^{2}\right) .
$$

One can easily check that:

$$
\begin{aligned}
\overline{u^{2}} & =\bar{u}^{2}+O\left(\varepsilon^{2}\right), \\
\overline{u \otimes u} & =\bar{u} \otimes \bar{u}+O\left(\varepsilon^{2}\right) .
\end{aligned}
$$


This is used in the sequel.

We also have the value of $p$ at the second order with Equation (2.9):

$$
p(t, x, z)=\frac{1}{F r^{2}}(h(t, x)-z)+\varepsilon \frac{\cos \theta}{R o} \int_{h}^{z} u_{1}+O\left(\varepsilon^{2}\right) .
$$

We replace it in the integrated momentum equation (2.10) and, using again the divergence free condition $(2.6 \mathrm{c})$ to express $w^{0}$ as a function of $h^{0}$ and $u^{0}$, we get

$$
\begin{aligned}
\partial_{t}\left(\boldsymbol{H}^{\mathbf{1}} \bar{u}\right) & +\operatorname{div}_{x}\left(\boldsymbol{H}^{\mathbf{1}} \bar{u} \otimes \bar{u}\right)+\frac{1}{2 F r^{2}} \nabla_{x}\left(\boldsymbol{H}^{\mathbf{1}}\right)^{2}-\varepsilon \frac{\cos \theta}{2 R o} \nabla_{x}\left(u_{1}^{0}\left(H^{0}\right)^{2}\right) \\
= & -K \boldsymbol{u}_{\left.\right|_{z=b} ^{\mathbf{1}}}-\nabla_{x} b\left(\frac{\boldsymbol{H}^{\mathbf{1}}}{F r^{2}}-\varepsilon \frac{\cos \theta}{R o} u_{1}^{0} H^{0}\right)+\varepsilon \operatorname{div}_{x}\left(H^{0} S_{x x}\right) \\
- & \frac{\sin \theta}{R o} \boldsymbol{H}^{\mathbf{1}} \bar{u}^{\perp}+\varepsilon \frac{\cos \theta}{2 R o}\left(H^{0}\right)^{2} e_{1} \operatorname{div}_{x} u^{0}-\varepsilon \frac{\cos \theta}{R o} H^{0} e_{1} \nabla_{x} b^{0} \cdot u^{0}+O\left(\varepsilon^{2}\right) .
\end{aligned}
$$

The velocity at the bottom $u_{\left.\right|_{z=b}}$ is given by

$$
\boldsymbol{u}_{\left.\right|_{z=b}}=\bar{u}\left(1+\frac{\varepsilon K}{\nu_{V}} \frac{H^{0}}{3}\right)^{-1}+O\left(\varepsilon^{2}\right)
$$

which leads to:

$$
\begin{aligned}
\partial_{t}\left(\boldsymbol{H}^{\mathbf{1}} \bar{u}\right) & +\operatorname{div}_{x}\left(\boldsymbol{H}^{\mathbf{1}} \bar{u} \otimes \bar{u}\right)+\frac{1}{2 F r^{2}} \nabla_{x}\left(\boldsymbol{H}^{\mathbf{1}}\right)^{2}=-K \bar{u}\left(1+\frac{\varepsilon K}{\nu_{V}} \frac{\boldsymbol{H}^{\mathbf{1}}}{3}\right)^{-1}-\varepsilon \nu_{H} \nabla_{x}\left(\boldsymbol{H}^{\mathbf{1}} \operatorname{div}_{x} \bar{u}\right) \\
& +2 \varepsilon \nu_{H} \operatorname{div}_{x}\left(\boldsymbol{H}^{\mathbf{1}} D_{x} \bar{u}\right)+\varepsilon \frac{\cos \theta}{2 R o} \nabla_{x}\left(\bar{u}_{1}\left(\boldsymbol{H}^{\mathbf{1}}\right)^{2}\right)+\varepsilon \frac{\cos \theta}{2 R o}\left(\boldsymbol{H}^{\mathbf{1}}\right)^{2} e_{1} \operatorname{div}_{x} \bar{u} \\
& -\varepsilon \frac{\cos \theta}{R o} \boldsymbol{H}^{\mathbf{1}} e_{1} \nabla_{x} b \cdot \bar{u}-\frac{\sin \theta}{R o} \boldsymbol{H}^{\mathbf{1}} \bar{u}^{\perp}-\nabla_{x} b\left(\frac{\boldsymbol{H}^{\mathbf{1}}}{F r^{2}}-\varepsilon \frac{\cos \theta}{R o} \bar{u}_{1} \boldsymbol{H}^{\mathbf{1}}\right)+O\left(\varepsilon^{2}\right) .
\end{aligned}
$$

Equations (2.15)-(2.16) form the Shallow Water System at the second order in nondimensional variables, with new cosine terms.

Finally, let us go back to the dimensional form to get the viscous Shallow Water System at the second order:

$$
\begin{aligned}
& \partial_{t} H+\operatorname{div}_{x}(H u)=0 \\
& \partial_{t}(H u)+\operatorname{div}_{x}(H u \otimes u)+\frac{g}{2} \nabla_{x} H^{2}=-\alpha_{0}(H) u-g H \nabla_{x} b \\
& \quad-\mu_{H} \nabla_{x}\left(H \operatorname{div}_{x} u\right)+2 \mu_{H} \operatorname{div}_{x}\left(H D_{x} u\right)+\Omega \cos \theta \nabla_{x}\left(u_{1} H^{2}\right) \\
& \quad+\Omega \cos \theta H^{2} e_{1} \operatorname{div}_{x} u-2 \Omega \sin \theta H u^{\perp}-2 \Omega \cos \theta H e_{1} \nabla_{x} b \cdot u+2 \Omega \cos \theta u_{1} H \nabla_{x} b
\end{aligned}
$$

where $\alpha_{0}(H)=k /\left(1+\frac{k H}{3 \mu_{V}}\right)$.

Conversely to the first order approximation (2.14), we now have viscous terms in the SW equations. The cosine part of the Coriolis force should be taken into account.

REMARK 1. If the latitude is not constant, the only difference with the previous development is that the term that reads $\Omega \cos \theta \nabla_{x}\left(u_{1} H^{2}\right)$ in the constant case must be replaced by $\Omega \nabla_{x}\left(\cos \theta u_{1} H^{2}\right)$ (with no additional difficulty), the other ones remaining unchanged.

REMARK 2. Note that, if we take the capillarity into account, the term $a H \nabla_{x} \Delta_{x} H+a H \nabla_{x} \Delta_{x} b$ adds to the right hand side of Equation (2.17b). 
2.2. Quasi-Geostrophic Equations. The Quasi-Geostrophic model is used for the abstract modeling of the ocean at mid-latitudes (see [5]). It is obtained from the Shallow Water System assuming that the Rossby and Froude numbers are very small.

Derivation of the Quasi-Geostrophic Equations with Cosine Effect. We consider the dimensionless Shallow Water System (2.15) - (2.16), that depends on the aspect ratio $\varepsilon$. We add in these equations a source term $\tilde{f}$ that could have been introduced in the previous derivation with no additional difficulty. In order to lighten the notations, we set $\mu=\mu_{H}$ and $\nu=\nu_{H}$ in the sequel.

We write an asymptotic development $u=u^{0}+\eta u^{1}+\ldots, H=1+\eta F H^{1}+\ldots$ where we suppose $R o=\eta, F r^{2}=F \eta^{2}, b=\eta \tilde{b}$, with $\eta \ll 1, F=\left(2 \Omega L_{\text {char }}\right)^{2} /\left(g H_{\text {char }}\right)$ and $\varepsilon$ fixed. Let us underline that, from now on, $\varepsilon$ is a fixed parameter, and the Rossby number $\eta$ is the asymptotic parameter, meant to go to zero. Doing this, we are not considering a crossed asymptotic $(\varepsilon, \eta) \rightarrow 0$, but we let $\eta$ go to zero as $\varepsilon$ remains fixed. In other words, refering to Figure 1.1, we are interested in path 2 - 4, that could be different from path $\star$. This is beyond the scope of this paper and is left to further studies. We also use the beta-plane approximation around the latitude $\theta_{0}$ (see [17]). We then have to study the following equations:

$$
\begin{aligned}
& \partial_{t} H+\operatorname{div}_{x}(H u)=0, \\
& \partial_{t}(H u)+\operatorname{div}_{x}(H u \otimes u)+\frac{1}{F \eta^{2}} H \nabla_{x} H=-\frac{\sin \theta_{0}}{\eta} H u^{\perp}-\beta x_{2} H u^{\perp} \\
& \quad+\varepsilon \frac{\cos \theta_{0}}{2 \eta} e_{1} H^{2} \operatorname{div}_{x} u-\frac{\varepsilon}{2} \tan \theta_{0} \beta x_{2} e_{1} H^{2} \operatorname{div}_{x} u+\varepsilon \frac{\cos \theta_{0}}{2 \eta} \nabla_{x}\left(H^{2} u_{1}\right) \\
& \quad-\frac{\varepsilon}{2} \tan \theta_{0} \beta \nabla_{x}\left(H^{2} u_{1} x_{2}\right)-\tilde{\alpha}_{0}(H) u+2 \nu \operatorname{div}_{x}(H D(u))-\nu \nabla_{x}(H \operatorname{div} u) \\
& \quad-\varepsilon \frac{\cos \theta_{0}}{\eta} H e_{1} \nabla_{x} b \cdot u+\varepsilon \tan \theta_{0} \beta x_{2} H e_{1} \nabla_{x} b \cdot u+\varepsilon \frac{\cos \theta_{0}}{\eta} u_{1} H \nabla_{x} b \\
& \quad-\varepsilon \tan \theta_{0} \beta x_{2} u_{1} H \nabla_{x} b-\frac{1}{F \eta^{2}} H \nabla_{x} b+H \tilde{f}+O(\eta) .
\end{aligned}
$$

At the first and second orders, Equation (2.18a) gives:

$$
\operatorname{div}_{x} u^{0}=0, \quad \text { and } \quad F \partial_{t} H^{1}+\operatorname{div}_{x} u^{1}+F \nabla_{x} H^{1} \cdot u^{0}=0 .
$$

We also look at the first and second orders of the momentum equation $(2.18 \mathrm{~b})$. The first order gives

$$
\nabla_{x} H^{1}+\left(\sin \theta_{0}-\varepsilon \frac{\cos \theta_{0}}{2} \partial_{x_{2}}\right) u^{0^{\perp}}+\frac{\nabla_{x} \tilde{b}}{F}=0 .
$$

Then we take the $\operatorname{curl}$ (i.e. $-\partial_{x_{2}}$ of the first component $+\partial_{x_{1}}$ of the second one) of the second order and get

$$
\begin{aligned}
\left(\partial_{t}+\right. & \left.u^{0} \cdot \nabla_{x}\right)\left(\operatorname{curl} u^{0}\right)=-\tilde{\alpha}_{0}(1) \operatorname{curl} u^{0}+\nu \Delta\left(\operatorname{curl} u^{0}\right)+\sin \theta_{0} F\left(\partial_{t} H^{1}+u^{0} \cdot \nabla_{x} H^{1}\right) \\
& -\sin \theta_{0} F u^{0} \cdot \nabla_{x} H^{1}-\beta u_{2}^{0}+\varepsilon F \frac{\cos \theta_{0}}{2} \partial_{x_{2}}\left(\partial_{t} H^{1}+u^{0} \cdot \nabla_{x} H^{1}\right) \\
& -\nabla_{x}^{\perp} H^{1} \cdot \nabla_{x} \tilde{b}+\varepsilon \cos \theta_{0} \partial_{x_{2}}\left(u^{0} \cdot \nabla_{x} \tilde{b}\right)+\varepsilon \cos \theta_{0} \nabla_{x}^{\perp} u_{1}^{0} \cdot \nabla_{x} \tilde{b}+\operatorname{curl} \tilde{f} .
\end{aligned}
$$

We note that

$$
\sin \theta_{0} u^{0} \cdot \nabla_{x} H^{1}-\varepsilon \frac{\cos \theta_{0}}{2}\left(\partial_{x_{2}} u^{0}\right) \cdot \nabla_{x} H^{1}+\nabla_{x}^{\perp} H^{1} \cdot \frac{\nabla_{x} \tilde{b}}{F}=0,
$$


and

$$
\left(\partial_{x_{2}} u^{0}\right) \cdot \nabla_{x} \tilde{b}+\nabla_{x}^{\perp} u_{1}^{0} \cdot \nabla_{x} \tilde{b}=0 .
$$

We define $\psi$ by $u^{0}=\nabla_{x}^{\perp} \psi$, and consequently $H^{1}=\left(\sin \theta_{0}-\varepsilon \cos \theta_{0} \partial_{x_{2}} / 2\right) \psi-\tilde{b} / F$, and find

$$
\begin{aligned}
\left(\partial_{t}+u^{0} \cdot \nabla_{x}\right) & \left(\left(\partial_{x_{1}}^{2}+\left(1+\varepsilon^{2} F \frac{\cos ^{2} \theta_{0}}{4}\right) \partial_{x_{2}}^{2}\right) \psi-\sin ^{2} \theta_{0} F \psi\right. \\
& \left.+\left(\sin \theta_{0}-\varepsilon \frac{\cos \theta_{0}}{2} \partial_{x_{2}}\right) \tilde{b}+\beta x_{2}\right)=-\tilde{\alpha}_{0}(1) \Delta \psi+\nu \Delta^{2} \psi+\operatorname{curl} \tilde{f}
\end{aligned}
$$

Equation (2.19) is the non-dimensional Quasi-Geostrophic Equation obtained from the viscous Shallow Water System at the second order with variable topography. The new terms (due to the Coriolis force) are $\varepsilon^{2} F \cos ^{2} \theta_{0} \partial_{x_{2}}^{2} \psi / 4$ and $\varepsilon \cos \theta_{0} \partial_{x_{2}} \tilde{b} / 2$. The "unusual" $\sin \theta_{0}$ coefficient is linked to an "unusual" Rossby number expression that arises in $F$ and $\tilde{b}$; in the "usual" case, the term $\sin \theta_{0}$ is replaced by 1 , and $\cos \theta_{0}$ by $1 / \tan \theta_{0}$.

Let us remove this problem coming back to dimensional variables. We get:

$$
\begin{array}{r}
D_{t}\left(\left(\partial_{x_{1}}^{2}+\left(1+\delta^{2}\right) \partial_{x_{2}}^{2}\right) \psi-\frac{\left(2 \Omega \sin \theta_{0}\right)^{2}}{g H_{\text {char }}} \psi+\left(1-\frac{H_{\text {char }}}{2 \tan \theta_{0}} \partial_{x_{2}}\right) \frac{2 \Omega \sin \theta_{0}}{H_{\text {char }}} b+\beta x_{2}\right) \\
=-\frac{1}{\varepsilon L_{\text {char }}} \alpha_{0}\left(H_{\text {char }}\right) \Delta \psi+\mu \Delta^{2} \psi+\operatorname{curl} f, \quad(2.20)
\end{array}
$$

where $D_{t}=\left(\partial_{t}+u^{0} \cdot \nabla_{x}\right), H_{\text {char }}=\varepsilon L_{\text {char }}$ and $\delta=\Omega \sqrt{H_{\text {char }} / g} \cos \theta_{0}$.

We add to this equation the following boundary conditions:

$$
\psi=0 \text { and } \Delta \psi=0 \text { on } \partial \mathcal{D},
$$

which respectively express the non-penetration condition and the slip condition.

REMARK 3. We can already notice that the cosine term has two different contributions. First, the laplacian is modified in the second direction by the small coefficient $\delta$. The other change is on the topography coefficient: we see the derivative of the topography in the second variable.

3. Mathematical Properties of the Quasi-Geostrophic Equations. The new terms in the $\mathrm{QG}$ model do not raise any additional mathematical difficulties. Hereafter, we proceed as in [3] to obtain a priori estimates that ensure well-posedness of the model (thanks to Galerkin method), and insist on the computations that are specific to the new model. The reader is referred to [3] for further details.

We consider a rectangular domain $\mathcal{D}$, multiply Equation (2.20) successively by $\psi$ and $\Delta \psi+\delta^{2} \partial_{x_{2}}^{2} \psi$, and integrate over $\mathcal{D}$. In the integrations by parts, the integrated term vanishes as we have the boundary conditions $\psi=0$ and $\Delta \psi=0$ on $\partial \mathcal{D}$.

To simplify the notations, we write $(2.20)$ as

$$
D_{t}\left(\left(\partial_{x_{1}}^{2}+\left(1+\delta^{2}\right) \partial_{x_{2}}^{2}\right) \psi-C_{H} \psi+B\left(x_{1}, x_{2}\right)+\beta x_{2}\right)=-\alpha \Delta \psi+\mu \Delta^{2} \psi+\operatorname{curl} f,
$$

or, if $J$ represents the jacobian operator,

$$
\begin{aligned}
\partial_{t}\left(\left(\Delta+\delta^{2} \partial_{x_{2}}^{2}\right) \psi-C_{H} \psi\right)+J\left(\psi,\left(\Delta+\delta^{2} \partial_{x_{2}}^{2}\right) \psi\right. & \left.-C_{H} \psi+B\left(x_{1}, x_{2}\right)+\beta x_{2}\right) \\
& =-\alpha \Delta \psi+\mu \Delta^{2} \psi+\operatorname{curl} f
\end{aligned}
$$


We obtain after multiplication of (3.1) by $\psi$ :

$$
\begin{gathered}
\int_{\mathcal{D}} \partial_{t}\left(\Delta \psi+\delta^{2} \partial_{x_{2}}^{2} \psi-C_{H} \psi\right) \cdot \psi=\alpha \int_{\mathcal{D}}(\nabla \psi)^{2}+\mu \int_{\mathcal{D}}(\Delta \psi)^{2}+\int_{\mathcal{D}} \operatorname{curl} f \cdot \psi, \\
\frac{d}{d t}\left(\|\nabla \psi\|_{L^{2}(\mathcal{D})}^{2}+\delta^{2}\left\|\partial_{x_{2}} \psi\right\|_{L^{2}(\mathcal{D})}^{2}+C_{H}\|\psi\|_{L^{2}(\mathcal{D})}^{2}\right)+2 \alpha \int_{\mathcal{D}}(\nabla \psi)^{2} \\
\quad+2 \mu \int_{\mathcal{D}}(\Delta \psi)^{2} \leq 2\left|\int_{\mathcal{D}} \operatorname{curl} f \cdot \psi\right| \leq \frac{C^{2}}{2 \alpha}\|\operatorname{curl} f\|_{L^{2}(\mathcal{D})}^{2}+\alpha\|\nabla \psi\|_{L^{2}(\mathcal{D})}^{2} .
\end{gathered}
$$

We finally get the inequality

$$
\begin{aligned}
\frac{d}{d t}\left(\|\nabla \psi\|_{L^{2}(\mathcal{D})}^{2}+\delta^{2}\left\|\partial_{x_{2}} \psi\right\|_{L^{2}(\mathcal{D})}^{2}+C_{H}\|\psi\|_{L^{2}(\mathcal{D})}^{2}\right) & +\alpha\|\nabla \psi\|_{L^{2}(\mathcal{D})}^{2} \\
& +2 \mu\|\Delta \psi\|_{L^{2}(\mathcal{D})}^{2} \leq \frac{C^{2}}{\alpha}\|\operatorname{curl} f\|_{L^{2}(\mathcal{D})}^{2},
\end{aligned}
$$

which ensure the following bounds:

$$
\nabla \psi \in L^{\infty}\left(0, T ;\left(L^{2}(\mathcal{D})\right)^{2}\right), \psi \in L^{\infty}\left(0, T ; L^{2}(\mathcal{D})\right), \Delta \psi \in L^{2}\left(0, T ; L^{2}(\mathcal{D})\right) .
$$

Similarly, multiplying (3.1) by $\Delta \psi+\delta^{2} \partial_{x_{2}} \psi$ we get:

$$
\begin{aligned}
\int_{\mathcal{D}} \partial_{t} & \left.\Delta \psi+\delta^{2} \partial_{x_{2}}^{2} \psi-C_{H} \psi\right)\left(\Delta \psi+\delta^{2} \partial_{x_{2}}^{2} \psi\right) \\
& +\int_{\mathcal{D}} J\left(\psi, \beta x_{2}+B\right)\left(\Delta \psi+\delta^{2} \partial_{x_{2}}^{2} \psi\right)=-\alpha \int_{\mathcal{D}} \Delta \psi\left(\Delta \psi+\delta^{2} \partial_{x_{2}}^{2} \psi\right) \\
& +\mu \int_{\mathcal{D}} \Delta^{2} \psi\left(\Delta \psi+\delta^{2} \partial_{x_{2}}^{2} \psi\right)+\int_{\mathcal{D}} \operatorname{curl} f\left(\Delta \psi+\delta^{2} \partial_{x_{2}}^{2} \psi\right)
\end{aligned}
$$

Let us write some properties of the different terms of this equality:

$$
\begin{aligned}
\int_{\mathcal{D}}\left(\Delta \psi+\delta^{2} \partial_{x_{2}}^{2} \psi\right)^{2} & =\|\Delta \psi\|_{L^{2}(\mathcal{D})}^{2}+\left\|\delta^{2} \partial_{x_{2}}^{2} \psi\right\|_{L^{2}(\mathcal{D})}^{2}+2 \delta^{2} \int_{\mathcal{D}} \Delta \psi \partial_{x_{2}}^{2} \psi \\
& =\|\Delta \psi\|_{L^{2}(\mathcal{D})}^{2}+\left\|\delta^{2} \partial_{x_{2}}^{2} \psi\right\|_{L^{2}(\mathcal{D})}^{2}+2 \delta^{2}\left\|\nabla \partial_{x_{2}} \psi\right\|_{L^{2}(\mathcal{D})}^{2}
\end{aligned}
$$

as the integrated term vanishes with the boundary conditions. We also have

$$
\begin{aligned}
& 2 \int_{\mathcal{D}} \Delta \psi \partial_{x_{2}}^{2} \psi \leq 2\|\Delta \psi\|_{L^{2}(\mathcal{D})}\left\|\partial_{x_{2}}^{2} \psi\right\|_{L^{2}(\mathcal{D})}, \\
& \left\|\partial_{x_{2}}^{2} \psi\right\|_{L^{2}(\mathcal{D})}^{2} \leq\left\|\partial_{x_{2}}^{2} \psi\right\|_{L^{2}(\mathcal{D})}^{2}+\left\|\partial_{x_{1}}^{2} \psi\right\|_{L^{2}(\mathcal{D})}^{2}+\left\|\partial_{x_{1}} \partial_{x_{2}} \psi\right\|_{L^{2}(\mathcal{D})}^{2}=\|\Delta \psi\|_{L^{2}(\mathcal{D})}^{2},
\end{aligned}
$$

and consequently

$$
\begin{aligned}
\int_{\mathcal{D}}\left(\Delta \psi+\delta^{2} \partial_{x_{2}}^{2} \psi\right)^{2} & \leq\left(1+2 \delta^{2}\right)\|\Delta \psi\|_{L^{2}(\mathcal{D})}^{2}+\delta^{4}\left\|\partial_{x_{2}}^{2} \psi\right\|_{L^{2}(\mathcal{D})}^{2} \\
& \leq\left(1+\delta^{2}\right)^{2}\|\Delta \psi\|_{L^{2}(\mathcal{D})}^{2} .
\end{aligned}
$$

In addition, one should note the equality:

$$
\begin{aligned}
\int_{\mathcal{D}} \Delta^{2} \psi\left(\Delta \psi+\delta^{2} \partial_{x_{2}}^{2} \psi\right) & =-\int_{\mathcal{D}}(\nabla \Delta \psi)^{2}+\delta^{2} \int_{\mathcal{D}} \Delta^{2} \psi \partial_{x_{2}}^{2} \psi \\
& =-\int_{\mathcal{D}}(\nabla \Delta \psi)^{2}-\delta^{2} \int_{\mathcal{D}}\left(\nabla \partial_{x_{2}}^{2} \psi\right)^{2}-\delta^{2} \int_{\mathcal{D}}\left(\nabla \partial_{x_{1}} \partial_{x_{2}} \psi\right)^{2}
\end{aligned}
$$


Finally we study the jacobian term:

$$
\begin{aligned}
\int_{\mathcal{D}} J\left(\psi, \beta x_{2}+B\right)\left(\Delta \psi+\delta^{2} \partial_{x_{2}}^{2} \psi\right) & \\
& \leq C\left(\beta+\|\nabla B\|_{L^{\infty}(\mathcal{D})}\right)\|\nabla \psi\|_{L^{2}(\mathcal{D})}\|\Delta \psi\|_{L^{2}(\mathcal{D})} .
\end{aligned}
$$

Gathering Inequalities (3.4) to (3.10), Equation (3.3) becomes:

$$
\begin{aligned}
\frac{d}{d t}(\| \Delta \psi+ & \left.\delta^{2} \partial_{x_{2}}^{2} \psi\left\|_{L^{2}(\mathcal{D})}^{2}+C_{H} \delta^{2}\right\| \partial_{x_{2}} \psi\left\|_{L^{2}(\mathcal{D})}^{2}+C_{H}\right\| \nabla \psi \|_{L^{2}(\mathcal{D})}^{2}\right) \\
& +2 \mu\|\nabla \Delta \psi\|_{L^{2}(\mathcal{D})}^{2}+2 \mu \delta^{2}\left(\left\|\nabla \partial_{x_{2}}^{2} \psi\right\|_{L^{2}(\mathcal{D})}^{2}+\left\|\nabla \partial_{x_{1}} \partial_{x_{2}} \psi\right\|_{L^{2}(\mathcal{D})}^{2}\right) \leq g(t)
\end{aligned}
$$

where $g$ reads

$$
\begin{aligned}
g(t)=C\left(\beta+\|\nabla B\|_{L^{\infty}(\mathcal{D})}\right)\|\nabla \psi\|_{L^{2}(\mathcal{D})} & \|\Delta \psi\|_{L^{2}(\mathcal{D})} \\
& +\alpha\|\Delta \psi\|_{L^{2}(\mathcal{D})}^{2}+\|\operatorname{curl} f\|_{L^{2}(\mathcal{D})}\|\Delta \psi\|_{L^{2}(\mathcal{D})} .
\end{aligned}
$$

Thanks to estimates $(3.2), g$ is in $L^{1}(0, T)$, which gives

$$
\begin{array}{ll}
\Delta \psi \in L^{\infty}\left(0, T ; L^{2}(\mathcal{D})\right), & \partial_{x_{2}}^{2} \psi \in L^{\infty}\left(0, T ; L^{2}(\mathcal{D})\right), \\
\nabla \Delta \psi \in L^{2}\left(0, T ;\left(L^{2}(\mathcal{D})\right)^{2}\right), & \nabla \partial_{x_{2}}^{2} \psi \in L^{2}\left(0, T ;\left(L^{2}(\mathcal{D})\right)^{2}\right), \\
\nabla \partial_{x_{1}} \partial_{x_{2}} \psi \in L^{2}\left(0, T ;\left(L^{2}(\mathcal{D})\right)^{2}\right) . &
\end{array}
$$

The proof of the existence of solutions to the Quasi-Geostrophic Equation (2.20) relies on Galerkin's method. We classically get the existence of solutions for the approximate problem (for detailed proof, the reader is referred to [3] and [7] for instance). We pass to the limit on the space dimension thanks to the previous a priori estimates and we get the following proposition:

THEOREM 3.1. If $\mathcal{D}$ is a rectangular domain, for all $f$ in $L^{2}\left(0, T ; L^{2}(\mathcal{D})\right)$, Equation (2.20) with the initial condition $\psi_{0}$ in $H^{3}(\mathcal{D}) \cap H_{0}^{1}(\mathcal{D})$ and the boundary conditions $\psi=0$ and $\Delta \psi=0$ on $\partial \mathcal{D}$ has a unique solution $\psi$ in $\mathcal{C}\left([0, T] ; H^{3}(\mathcal{D}) \cap H_{0}^{1}(\mathcal{D})\right) \cap$ $L^{2}\left(0, T ; H^{4}(\mathcal{D}) \cap H_{0}^{1}(\mathcal{D})\right)$.

4. Numerical Results: Cosine Effects on Large Scale Computations. We now present numerical results for the QG equations and show that the cosine effect cannot be neglected. These simulations are performed using finite differences in space, with an explicit Leap-Frog scheme in time. One step every hundred, the LeapFrog step is replaced by an Euler explicit one, in order to avoid stability problems. To compare the numerical simulations of the QG model with and without the new terms, we use and modify an existing solver proposed by [18].

4.1. Methodology. Equation (2.20) can be rewritten in a more "physical" way, that is:

$$
\begin{gathered}
\frac{\partial \phi}{\partial t}+J\left(\psi, \phi+\left(\operatorname{Id}-\frac{H_{\text {char }}}{2 \tan \theta_{0}} \partial_{x_{2}}\right) \frac{2 \Omega \sin \theta_{0}}{H_{\text {char }}} b+\beta x_{2}\right) \\
=-\frac{1}{\varepsilon L_{\text {car }}} \alpha_{0}\left(H_{\text {car }}\right) \Delta \psi+\mu \Delta^{2} \psi+\operatorname{curl} f
\end{gathered}
$$

where $J$ is the jacobian, and $\phi$ a potential vorticity which reads

$$
\phi=\left(\Delta+\delta^{2} \partial_{x_{2}}^{2}-\frac{\left(2 \Omega \sin \theta_{0}\right)^{2}}{g H_{\text {char }}} \mathrm{Id}\right) \psi
$$


The scheme used to solve the Quasi-Geostrophic Equation is the following: suppose that we know all the quantities at time $t_{n}, \phi^{n}, \psi^{n}$ and the diverse coefficients. Then we compute the term $f l^{n}$ which represents the time derivative of $\phi$ at $t_{n}$, namely:

$$
\begin{aligned}
f l^{n}=J\left(\phi^{n}+\left(\operatorname{Id}-\frac{H_{\text {char }}}{2 \tan \theta_{0}} \partial_{x_{2}}\right)\right. & \left.\frac{2 \Omega \sin \theta_{0}}{H_{\text {char }}} b+\beta x_{2}, \psi^{n}\right) \\
& -\frac{1}{\varepsilon L_{\text {car }}} \alpha_{0}\left(H_{\text {car }}\right) \Delta \psi^{n}+\mu \Delta^{2} \psi^{n}+\operatorname{curl} f^{n} .
\end{aligned}
$$

We thus get $\phi$ at time $t_{n+1}$ and obtain $\psi^{n+1}$ thanks to Equation (4.1). If we start with an initial condition identically equal to zero for example, we are able to plot the stream function at any time.

The principal difficulty of the numerical scheme is the resolution of the equation that gives $\phi^{n+1}$ as a function of $\psi^{n+1}$. Note that if $\delta=0$, we are led to solve a Poisson equation. The methodology we used is the one developed in [22], that is a combination of symmetries and fast Fourier transforms. More precisely, as we have Dirichlet boundary conditions, we define two quantities $\zeta^{n+1}$ and $\xi^{n+1}$ by $\left[0 ; \phi^{n+1} ; 0 ; \phi_{\sigma}^{n+1}\right]$ (resp. $\left[0 ; \psi^{n+1} ; 0 ; \psi_{\sigma}^{n+1}\right]$ ) where $f_{\sigma}$ is the symmetric vector of $(-f)$ in the first variable $x_{1}$. We compute the Fourier transforms $\hat{\zeta}$ and $\hat{\xi}$ of $\zeta$ and $\xi$ in the $x_{1}$ direction and we get a system in $x_{2}$. The matrix $M$ of this system $(M \hat{\xi}=\hat{\zeta})$ is tridiagonal and consequently can easily be inverted. The last step is to calculate the inverse of the Fourier transform to get $\psi^{n+1}$ from $\phi^{n+1}$.

4.2. Choice of the Parameters. We consider that $\mathcal{D}$ is a square basin of length $L=4.000 \mathrm{~km}$, which is $5.000 \mathrm{~m}$ deep with a $100 \times 100$ points grid, and thus the aspect ratio is $\varepsilon=1.25 \times 10^{-3}$. We borrow the other physical parameters from [10]. We check that these parameters match the case studied at the beginning: in particular, the nondimensional horizontal viscosity is of order of the aspect ratio. We choose the forcing term (wind) as $-10^{-2} \sin \left(2 \pi x_{2} / L\right)$ and let the model run over nearly 1600 years (note that we have $T=O\left(\varepsilon^{-1}\right)$ ) to ensure that the convergence error is small compared to

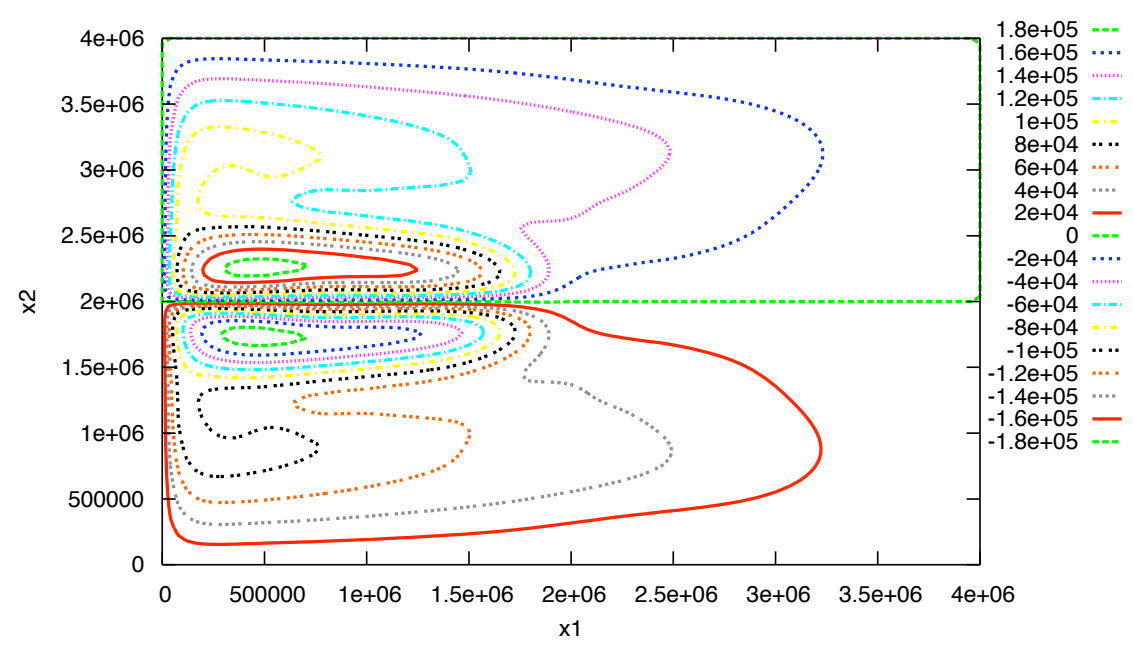

FIG. 4.1. Time-average of the stream function over 1600 years. The upper part is negative so the velocity is in the counterclockwise direction. 
the cosine effect. We then compare the results obtained with and without the cosine terms, with a flat or a varying bottom. A first look at the evolution of the energy behaviour indicates that the system is chaotic; we thus present the time-average of $\psi$ instead of $\psi$ in the following.

4.3. Flat Bottom. As mentionned above, the cosine effect has two correlated contributions. In order to disconnect these terms, we first consider a flat bottom so that the second term (depending on the topography variations) vanishes. Figure 4.1 shows the stream function without the cosine effect, and we plot in Figure 4.2 the difference between the two models: this corresponds to the numerical contribution of the cosine effet.

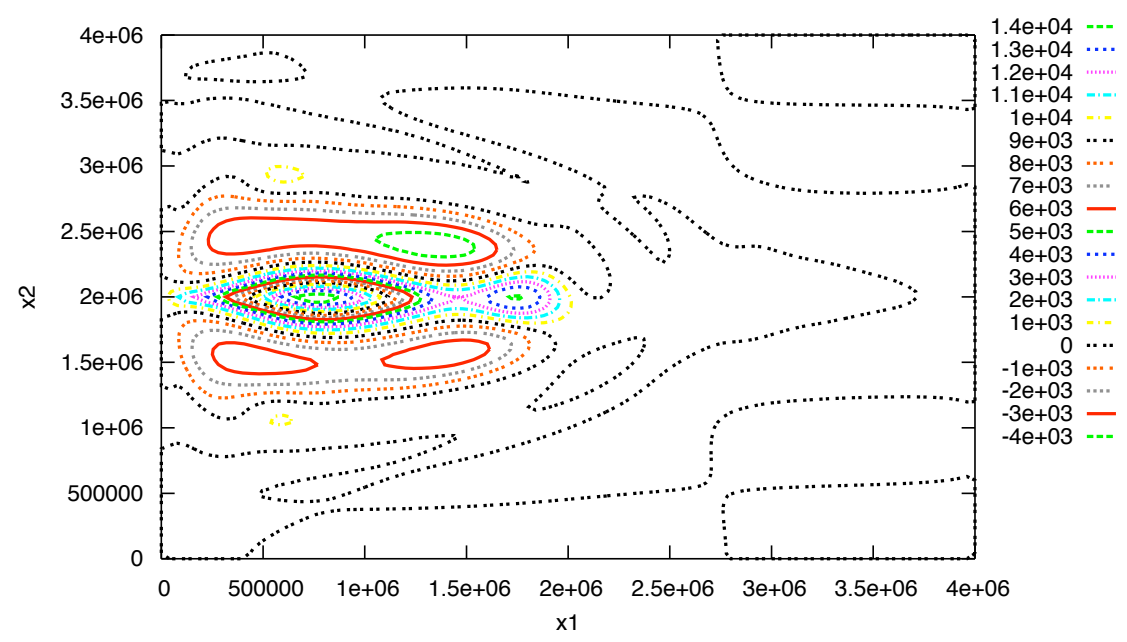

FIG. 4.2. Time-average of the difference between the stream function with the cosine terms and the stream function without these terms, over 1600 years.

The difference is mainly located in the jet, with a maximum value of $1.4 * 10^{4}$. Comparing this to Figure 4.1 where the maximum value is $1.4 * 10^{5}$, we show that the contribution of the new model is about ten percents.

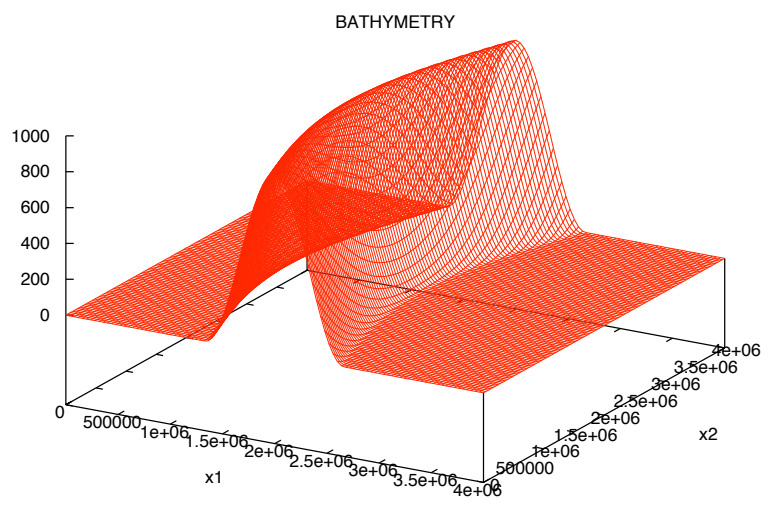

FIG. 4.3. An example of bottom. 
4.4. Varying Bottom. Let us now consider the cosine effect, with a bottom depending on the second variable $x_{2}$. We use a topography roughly resembling to the Mid-Atlantic ridge, as can be seen in Figure 4.3 .

As for the flat bottom, we present in Figure 4.4 the time-average of the stream function, solution of the QG model without any cosine effect. Figure 4.5 plots the difference between the two models, with and without the cosine terms.

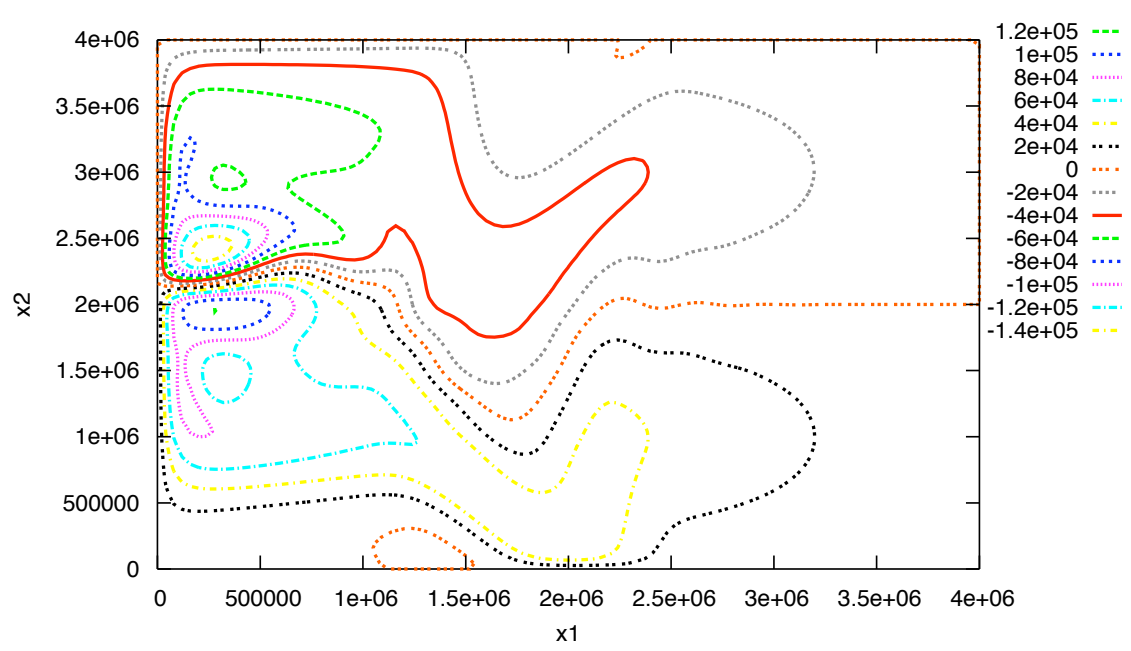

FiG. 4.4. Time-average of the stream function over 1600 years with a varying bottom. The upper part is negative so the velocity is in the counterclockwise direction.

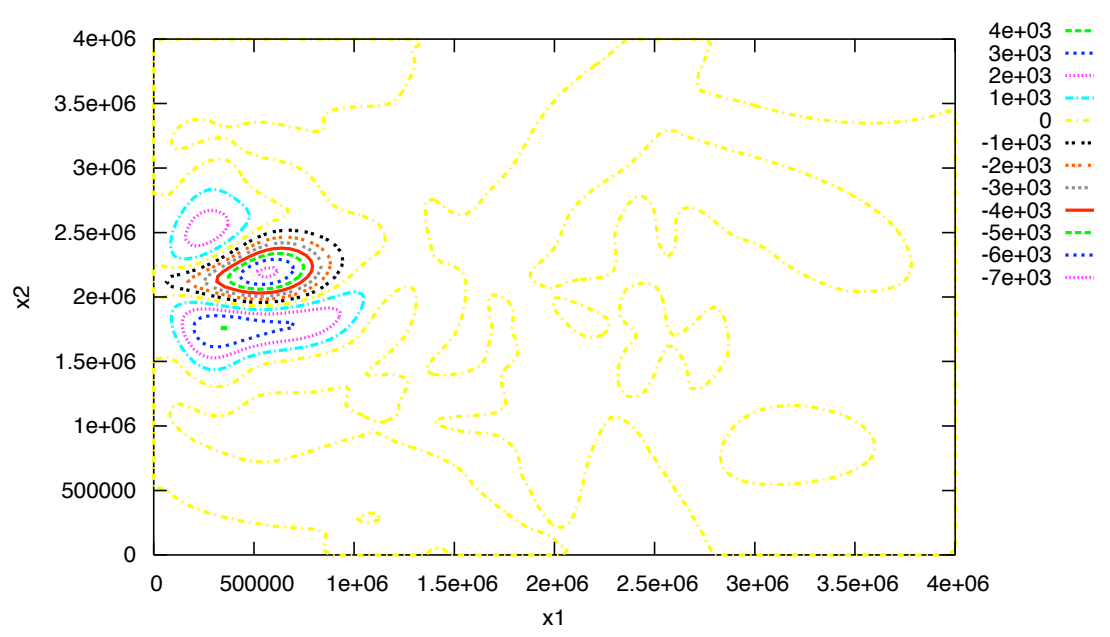

FIG. 4.5. Time-average of the difference between the stream function with the cosine terms and the stream function without these terms, over 1600 years, with a varying bottom.

In this configuration, the contribution of the cosine effect is weaker: only a few percents. 
5. Conclusion. We derived new SW and QG models from NSE with anisotropic turbulent viscosities. We proved that, when the horizontal viscosity is of the order of the aspect ratio (which is physically reasonnable), some new terms appear in the derivation of the viscous Shallow Water Equations. After providing some new theoretical results on the corresponding model, we perform numerical experiments that confirm that the so-called cosine effect has to be taken into account.

Acknowledgments. The authors are grateful to E. Kazantsev, C. Kazantsev and D. Bresch for very fruitful discussions related to this article.

\section{REFERENCES}

[1] E. Audusse. A multilayer Saint-Venant model: derivation and numerical validation. Discrete Contin. Dyn. Syst. Ser. B, 5(2):189-214, 2005.

[2] P. Azérad and F. Guillén. Mathematical justification of the hydrostatic approximation in the primitive equations of geophysical fluid dynamics. SIAM J. Math. Anal., 33(4):847-859 (electronic), 2001.

[3] C. Bernier. Étude et parallélisation d'un code d'éléments finis pour la modélisation quasigéostrophique des circulations océaniques. Phd, Institut National Polytechnique de Grenoble, 1990.

[4] P. Bougeault and R. Sadourny. Dynamique de l'atmosphère et de l'océan, chapter 5, pages 115-118. Éditions de l'École Polytechnique, 2001.

[5] A. Bourgeois and J. T. Beale. Validity of the quasigeostrophic model for large-scale flow in the atmosphere and ocean. SIAM J. Appl. Math., 25(4):1023-1068, 1994.

[6] D. Bresch and B. Desjardins. Existence of global weak solutions for a $2 \mathrm{D}$ viscous shallow water equations and convergence to the quasi-geostrophic model. Comm. Math. Phys., 238(1-2):211-223, 2003.

[7] H. Brézis. Analyse fonctionnelle, théorie et applications. Dunod, 2000.

[8] F. Dumas and P. Lazure. An external-internal mode coupling for a 3d hydrodynamical model for applications at regional scale (mars). Advances in Water Resources, 2007. to appear.

[9] J.-F. Gerbeau and B. Perthame. Derivation of viscous Saint-Venant system for laminar shallow water; numerical validation. Discrete and Continuous Dynamical Systems-series B., 1(1):89-102, 2001.

[10] A. E. Gill. Athmosphere-Ocean Dynamics. New York: Academic Press, 1982.

[11] E. Grenier and N. Masmoudi. Ekman layers of rotating fluids, the case of well prepared initial data. Comm. Partial Diff. Equations, 22:953-975, 1997.

[12] C. D. Levermore and M. Sammartino. A shallow water model with eddy viscosity for basins with varying bottom topography. Nonlinearity, 14:1493-1515, 2001.

[13] C. Lucas. Effet cosinus sur un modèle visqueux de type saint-venant et ses équations limites de type quasi-géostrophique et lacs. C. R. Acad. Sci. Paris, Ser. I, 345:313-318, 2007.

[14] G. Madec. NEMO reference manual, ocean dynamics component : NEMO-OPA, volume 27. Note du Pole de modélisation, Institut Pierre-Simon Laplace (IPSL), 2008.

[15] F. Marche. Theoretical and numerical study of shallow water models ; applications to nearshore hydrodynamics. Phd, Université Bordeaux 1, 2005.

[16] N. Masmoudi. Ekman layers of rotating fluids: The case of general initial data. Comm. Pure Appl. Math., 53(4):432-483, 2000.

[17] J. Pedlosky. Geophysical fluid dynamics, 2nd edition. Springer, 1987.

[18] C. Le Provost, C. Bernier, and E. Blayo. A comparison of two numerical methods for integrating a quasi geostrophic multilayer model of ocean circulations. J. Comp. Phys., 110:341-359, 1994.

[19] A. Rousseau, R. Temam, and J. Tribbia. Boundary value problems for the inviscid primitive equations in limited domains. To appear in Handbook of Numerical Analysis, 2008.

[20] A. J.-C. de Saint-Venant. Théorie du mouvement non-permanent des eaux, avec application aux crues des rivières et à l'introduction des marées dans leur lit. Comptes Rendus de l'Académie des Sciences, 73:147-154, 1871.

[21] E. Simonnet, T. Tachim Medjo, and R. Temam. On the order of magnitude of the baroclinic flow in the primitive equations of the ocean. Ann. Mat. Pura Appl. (4), 185(suppl.):S293-S313, 2006. 
[22] A. Wiegmann. Fast elliptic solvers on rectangular parallelepipeds. http://www.itwm.fhg.de/sks/employees/wiegmann/References/fft.pdf. 